\title{
Significados sobre la derivada evidenciados por alumnos de carreras de Ingeniería en una universidad mexicana
}

\author{
Víctor Larios Osorio, Universidad Autónoma de Querétaro (México) \\ Rosa Elvira Páez Murillo, Universidad Autónoma de la Ciudad de México (México) \\ Hugo Moreno Reyes, Centro Interdisciplinario de Investigación y Docencia en Educación \\ Técnica (México)
}

\begin{abstract}
Significados sobre la derivada evidenciados por alumnos de carreras de Ingeniería en una universidad mexicana

\section{Resumen}

El objetivo de este artículo es responder la pregunta: ¿Cuál es el significado del objeto matemático derivada para un grupo de futuros ingenieros? Se usa como referente teórico el Enfoque Ontosemiótico del Conocimiento y la Instrucción Matemáticos (EOS). En la metodología se considera una reconstrucción de los significados parciales de la Derivada para el diseño y aplicación de un cuestionario con ocho tareas para identificar el uso de esos significados parciales y, utilizando el método de análisis ontosemiótico, se analizaron las respuestas de los participantes. Con los resultados se concluye que el significado parcial predominante está vinculado con la idea de recta tangente a la gráfica de la función original y se observó la preferencia por el uso del registro gráfico en lugar del tabular. Se cierra con algunas reflexiones sobre las tareas que deben contemplarse en los cursos de Cálculo dirigidos a la formación de ingenieros.
\end{abstract}

Palabras clave. Derivada; significados parciales; formación de ingenieros; enseñanza del cálculo.

Meanings on the derivative evidenced by Engineering students at a Mexican university

Abstract

This paper's goal is to answer the question: What is the meaning of the mathematical object Derivative for a group of future engineers? The Ontosemiotic Approach to Mathematical Cognition and Instruction (OSA) is used as theoretical reference. The methodology considers a reconstruction of the Derivative's partial meanings for the design, and application of a questionnaire with eight tasks to identify the use of these partial meanings. The participants' responses were analyzed using the ontosemiotic analysis method. We conclude that the predominant partial meaning is linked to the idea of a tangent line to the graph of the original function, with the observation of the preference for the use of the graphic register instead of the tabular one. We close the paper with some reflections on the tasks that Calculus courses specialized for engineers should consider.

Keywords. Derivative; partial meanings; engineering education; calculus' teaching.

\section{Introducción}

En el área de Educación Matemática se ha considerado relevante investigar sobre las conexiones matemáticas y se ha generado un cierto consenso sobre su fomento en los procesos de instrucción de esta materia. La relevancia que se otorga al establecimiento de conexiones matemáticas está relacionada con la importancia que se da a la reflexión sobre la complejidad de los objetos matemáticos (y en su articulación) en algunos de los marcos teóricos del área. En el Enfoque Ontosemiótico de la Cognición y la Instrucción Matemáticas (EOS) (Godino et al., 2019) se modeliza la complejidad de los objetos matemáticos por medio de significados parciales y se considera que el estudio de los diferentes significados de un objeto matemático es un tema relevante de 
investigación. En este enfoque, los significados parciales se modelizan en términos de prácticas matemáticas y de las configuraciones de objetos primarios que las activan. Para la realización de una práctica matemática y para la interpretación de sus resultados como satisfactorios se necesita poner en funcionamiento objetos matemáticos. Si consideramos, por ejemplo, los objetos matemáticos que participan en la realización y evaluación de la práctica que permite resolver una situación-problema vemos el uso de lenguajes verbales, simbólicos, etc. Estos lenguajes son la parte ostensiva de una serie de conceptos, proposiciones y procedimientos que intervienen en la elaboración de argumentos para decidir si las acciones simples que componen la práctica matemática son satisfactorias. Así, cuando se realiza y evalúa una práctica matemática, se activa un conjunto formado por problemas, notaciones, definiciones, proposiciones, procedimientos y argumentos.

En relación con el cálculo en las carreras de Ingeniería, la expectativa es que sirva como base para un desarrollo académico y escolar apropiado, como también para un desempeño laboral tras el egreso del estudiante de la universidad. Por tanto, se espera que se cumpla con lo señalado por Gnedenko y Khalil (1979) en el sentido de que las matemáticas se convierten para los ingenieros en un método potente, flexible y útil de interpretar fenómenos, particularmente los relacionados con las ciencias, la ingeniería y la industria. Se trata de que los ingenieros sean competentes en el uso de matemáticas para la resolución de problemas de su profesión; en consecuencia, es necesario que los profesores de matemáticas en la carrera de ingeniería tengan en cuenta, en sus procesos de instrucción, la complejidad del objeto matemático que se pretende enseñar.

Dada esta importancia que se ha dado al cálculo en la formación de ingenieros, se ha propiciado un interés por estudiar su aprendizaje en ese contexto (e. g. Bingolbali et al., 2007; Cuevas et al., 2018; Gnedenko y Khalil, 1979; Pino-Fan et al., 2018; RomoVázquez, 2014).

Artigue et al. (2007) presentan resultados sobre las diferencias en las formas de concebir las matemáticas por parte de estudiantes de Matemáticas y los de Ingeniería. Bingolbali et al. (2007) abordan una línea similar de investigación y muestran que los estudiantes de Ingeniería usan la derivada vinculada a la noción de cambio, es decir, una concepción física de movimiento, mientras que, para el caso de carreras de Matemáticas, el énfasis se pone en una relación gráfica, no física, vinculada con la pendiente de la recta tangente. En un contexto longitudinal entre niveles educativos, Ariza y Llinares (2009) reportan que las preferencias en el uso de los distintos registros de representación semiótica son diferentes dependiendo del nivel educativo (bachillerato o licenciatura), aunque reconocen la necesidad del buen manejo de varios registros para tener mayor grado de comprensión del concepto.

Pino-Fan et al. (2018) se han centrado en el estudio de los significados que los estudiantes de Ingeniería le asignan a la antiderivada a fin de identificar significados parciales del objeto matemático que emergen en sus prácticas matemáticas. El interés de identificar estos significados parciales proviene del hecho de que los objetos matemáticos tienen un significado holístico compuesto por un conjunto de significados parciales desde el punto de vista histórico. Pino-Fan et al. (2011) hacen un estudio histórico de la evolución de la noción de derivada e identifican nueve significados parciales, que van desde la idea de recta tangente en la Geometría griega, hasta la definición formal basada en la noción de límite, pasando por las ideas de variación propuestas por Newton o vinculadas al estudio de los fenómenos físicos. Se propone que el significa- 
do holístico del objeto matemático derivada viene a ser la conjunción de los nueve significados parciales identificados.

Cada uno de los significados parciales está vinculado a ciertas ideas y nociones existentes en el contexto científico y filosófico en el cual se desarrolla, porque en su momento respondió a sistemas de prácticas específicos que pertenecieron a las personas que participaron en su desarrollo dentro de contextos específicos. Además, estos significados parciales pueden privilegiar el uso de ciertos registros de representación semióticos (Duval, 1993) por encima de otros. Esto toma relevancia no sólo porque cada estudiante tiene sus prácticas matemáticas al momento de abordar el aprendizaje de un objeto matemático, las cuales son producto de su misma formación académica y contexto personal, sino porque algunos de estos significados parciales tienen mayor o menor relevancia, por los sistemas de prácticas involucradas en su desarrollo, en la formación y desenvolvimiento de una cierta comunidad de personas que las aplica, que en este caso sería la comunidad de ingenieros.

Existen propuestas en la literatura actual orientadas específicamente al estudio del cálculo considerando las prácticas matemáticas ajustadas a la comunidad de ingenieros. Este es el caso de propuestas como Arcos (2019), que privilegian el uso de los infinitesimales, ya que considera que la aproximación basada en límites es laboriosa, poco intuitiva y alejada de la realidad, aunque sea la más utilizada actualmente (Arcos y Sepúlveda, 2014). Pino-Fan et al. (2013), al realizar un estudio sobre los significados de la derivada que pretende el currículo de bachillerato en México, identificaron que sólo se movilizaba el significado parcial vinculado a la noción de límite de cociente de incrementos, el último en el desarrollo histórico. Orts et al. (2016) analizaron libros de texto del bachillerato español y reportaron que no se hace explícito el paso entre los tipos de concepción de la derivada que se han desarrollado a lo largo de la historia de las matemáticas.

Lo anterior entra en conflicto con las observaciones de Hitt (2005), quien hace hincapié en promover la visualización matemática utilizando diferentes representaciones, lo cual "impulsará a los estudiantes a un nivel más profundo de los conceptos propios del cálculo" (p. 106). En este sentido, está de acuerdo en que se desarrollen materiales que consideren apropiadamente herramientas digitales que permitan no sólo las representaciones algebraicas, sino representaciones gráficas.

Así pues, en general la Derivada, en los procesos de formación de ingenieros, ha sido centro de atención de estudios de tipo cognitivo e instruccional (Artigue et al,, 2007). En estos estudios ha quedado en evidencia las dificultades que existen en su enseñanza y en su aprendizaje.

El objetivo de esta investigación es responder la pregunta: ¿Cuál es el significado del objeto matemático derivada para un grupo de futuros ingenieros? Con esto se busca aportar información académica orientada a cambios curriculares que consideren los significados que tienen y desarrollan los alumnos para considerar lo que ellos mismos requieren para su práctica futura como ingenieros.

\section{Marco teórico}

El significado de los objetos matemáticos se concibe desde una perspectiva pragmática-antropológica que considera la relatividad del contexto en el cual son utilizados. Es decir, el significado de un objeto matemático puede ser definido como el sistema de prácticas operativas y discursivas que una persona (o una comunidad, denominada "institución") lleva a cabo para resolver cierto tipo de problemas en los cuales 
los objetos considerados intervienen (Godino et al., 2007). Con base en esto se pueden considerar los significados de un objeto matemático desde dos perspectivas: la institucional y la personal. En el EOS se recurre a la noción de configuración epistémica que permite un análisis más preciso de los objetos matemáticos primarios y su red de relaciones: problemas, elementos de lenguaje, definiciones, proposiciones, procedimientos y argumentos. Todo esto desde el punto de vista de la institución (comunidad) de referencia que es la matemática. Ahora bien, cuando se consideran estos objetos y su red de relaciones desde el punto de vista de los individuos se habla de configuraciones cognitivas. Para el análisis que se presentará, se ha tomado la noción de configuración cognitiva de los estudiantes de Ingeniería.

Se ha considerado Pino-Fan et al. (2011), un estudio ontológico e histórico del concepto de derivada con nueve configuraciones epistémicas que consideran significados parciales del significado holístico del objeto derivada. Estas configuraciones están vinculadas con sus respectivos contextos histórico-filosóficos y son: 1) prácticas para encontrar rectas tangentes en la matemática; 2) estudios sobre la variación en la edad media; 3) el uso de métodos algebraicos para hallar rectas tangentes a curvas; 4) concepciones cinemáticas para el trazado de tangentes; 5) las ideas intuitivas de límite para el cálculo de máximos y mínimos; 6) el uso de métodos infinitesimales en el cálculo de tangentes; 7) el cálculo de fluxiones; 8) el cálculo de diferencias; y 9) la concepción de derivada como límite. A su vez, por la "afinidad" en el tipo de prácticas matemáticas involucradas, las clasificaron en tres tipos de configuraciones epistémicas: A) tangentes (1, 3, 6 y 8); B) variaciones y velocidades (2, 4 y 7); y C) límites (5 y 9). Se observa cómo el nivel de complejidad en términos semióticos y de abstracción aumenta conforme se avanza en el desarrollo histórico.

\section{Aspectos metodológicos}

En el desarrollo y presentación de la investigación, se consideran los siguientes pasos: 1) se exhibe una reconstrucción del significado holístico del objeto matemático derivada, concretado en nueve significados parciales; 2) se diseña un cuestionario con tareas mediadoras de significados; y 3) se aplica el cuestionario y se analizan de manera cualitativa y cuantitativa las respuestas de participantes. Primó, sin embargo, una metodología cualitativa, dado que es estudio exploratorio orientado a la observación de variables cualitativas, aunque se utiliza estadística descriptiva para complementar con un análisis de porcentajes de variables cuantitativas como es un grado de exactitud.

\subsection{El cuestionario}

El cuestionario fue diseñado para identificar los significados usados por los estudiantes sobre la Derivada a través de sus prácticas matemáticas. El cuestionario fue aplicado en una sesión de dos horas y está conformado por ocho tareas, las cuales identificamos con la letra $\mathrm{T}$ y el número respectivo (algunas incluyen subtareas). Estas tareas están relacionadas con los significados parciales primarios de la derivada que Pino-Fan et al. (2011) identificaron a partir del estudio histórico-epistemológico en el que muestran el significado holístico de referencia de la derivada. La Tabla 1 muestra las características generales de cada tarea. 
Tabla 1. Resumen de características de las tareas del cuestionario

\begin{tabular}{|c|c|c|c|}
\hline Tarea & $\begin{array}{c}\text { Práctica matemática } \\
\text { esperada }\end{array}$ & $\begin{array}{c}\text { Representación } \\
\text { activada }\end{array}$ & $\begin{array}{c}\text { Significado } \\
\text { parcial activado } \\
\end{array}$ \\
\hline $\begin{array}{l}\text { T1: Significado de derivada } \\
\text { en un punto }\end{array}$ & $\begin{array}{l}\text { Descripción de significados } \\
\text { personales de derivada en un } \\
\text { punto }\end{array}$ & Verbal/escrita & Holístico \\
\hline $\begin{array}{l}\text { T2: Significado de derivada } \\
\text { como función }\end{array}$ & $\begin{array}{c}\text { Descripción de significados } \\
\text { personales de función } \\
\text { derivada }\end{array}$ & Verbal/escrita & Holístico \\
\hline $\begin{array}{l}\text { T3: Cálculo de derivada de } \\
\text { una función en un punto a } \\
\text { partir de una gráfica }\end{array}$ & $\begin{array}{c}\text { Relación entre pendiente de } \\
\text { la recta tangente a la gráfica } \\
\text { de la función en un punto y } \\
\text { derivada }\end{array}$ & $\begin{array}{l}\text { Gráfica y } \\
\text { simbólica }\end{array}$ & Tangentes \\
\hline $\begin{array}{l}\text { T4: Estimación de derivada } \\
\text { de una función a partir de } \\
\text { una tabulación }\end{array}$ & $\begin{array}{l}\text { Tratamiento de derivada } \\
\text { como razón de cambio en } \\
\text { representación tabular de } \\
\text { una función }\end{array}$ & $\begin{array}{l}\text { Tabular y } \\
\text { simbólica }\end{array}$ & $\begin{array}{l}\text { Variación/Razón } \\
\text { de cambio }\end{array}$ \\
\hline $\begin{array}{l}\text { T5: Identificación de punto } \\
\text { crítico y su relación con la } \\
\text { recta tangente }\end{array}$ & $\begin{array}{l}\text { Relación entre recta tangente } \\
\text { horizontal y existencia de un } \\
\text { punto crítico en la curva }\end{array}$ & Simbólica & $\begin{array}{l}\text { Tangente/ } \\
\text { Máximos y } \\
\text { mínimos }\end{array}$ \\
\hline $\begin{array}{l}\text { T6: Identificación de } \\
\text { variaciones de la función } \\
\text { derivada a partir de la } \\
\text { representación gráfica de la } \\
\text { función original }\end{array}$ & $\begin{array}{l}\text { Identificación de } \\
\text { propiedades de la función } \\
\text { derivada a partir de } \\
\text { comportamiento de la } \\
\text { gráfica de una función }\end{array}$ & Gráfica & $\begin{array}{l}\text { Variación/ } \\
\text { Máximos y } \\
\text { mínimos }\end{array}$ \\
\hline $\begin{array}{l}\text { T7: Representación de } \\
\text { función derivada a partir de } \\
\text { la gráfica de una función a } \\
\text { trozos }\end{array}$ & $\begin{array}{l}\text { Relación de variación de una } \\
\text { función a trozos con } \\
\text { representación de la función } \\
\text { derivada }\end{array}$ & $\begin{array}{l}\text { Gráfica y } \\
\text { simbólica }\end{array}$ & Tangente/Límites \\
\hline $\begin{array}{l}\text { T8: Identificación de caso } \\
\text { en que no es derivable la } \\
\text { función y la relación con la } \\
\text { recta tangente }\end{array}$ & $\begin{array}{l}\text { Relación entre valor donde } \\
\text { hay un "pico" en la gráfica y } \\
\text { existencia de recta tangente }\end{array}$ & $\begin{array}{l}\text { Gráfica y } \\
\text { simbólica }\end{array}$ & Tangente/Límites \\
\hline
\end{tabular}

\subsection{Participantes y contexto}

El cuestionario se aplicó a una muestra por conveniencia de estudiantes de Ingeniería de tres grupos de una institución superior pública en México. El primer grupo constó de 26 estudiantes, el segundo de 39 y el tercero de 25 , por lo que en total 90 estudiantes participaron. El requisito esencial fue que al momento de la recolección de la información ya hubiesen cursado la asignatura de cálculo diferencial.

Para determinar si los tres grupos se comportaron de manera similar al contestar, se compararon las respuestas correctas, parcialmente correctas e incorrectas en cada grupo y se aplicó una prueba de hipótesis de análisis de varianza (ANOVA). Encontramos que, con un grado de confianza de $95 \%$, no hay diferencias significativas en los grupos de estudiantes de Ingeniería que participaron. En consecuencia, los 90 estudiantes fueron considerados como una sola muestra.

Al momento de la aplicación del instrumento no se proporcionó información u orientación adicional a los participantes en cuanto el contenido del cuestionario y sus respuestas. Durante la aplicación no hubo interacción con el profesor de los cursos. 


\section{Resultados}

En esta sección se presenta el análisis de los resultados de seis tareas del cuestionario. Omitimos el análisis de la T5 y de la T8, dado que no se obtuvo mayor información por la gran cantidad de respuestas incorrectas y/o abstención de respuestas, lo cual indica un nivel de complejidad de estas tareas.

\subsection{T1 y T2: Significados de derivada en un punto y como función}

Por las similitudes de ambas tareas, su análisis se hace de manera conjunta, pues en ambos casos la intención fue que los alumnos expresaran sus significados personales con la diferencia de que, en la primera tarea, el foco era para el caso particular de un valor del dominio (derivada en un punto) y, en la segunda, se orientó a la generalización de todos los valores del dominio (derivada como función), como se puede evidenciar en el enunciado específico de la tarea:

T1: ¿Qué es para ti la derivada de una función en un punto?

T2: ¿Qué es para ti la función derivada?

Dentro de los resultados tenemos que el $35 \%$ de las respuestas fueron consideradas erróneas, el $45 \%$ fueron parcialmente correctas y sólo el $25 \%$ se consideraron correctas (en el porcentaje general de las dos tareas, pero prácticamente de manera similar en cada una de ellas). En la Tabla 2 aparece una caracterización de las respuestas provistas por los alumnos, en términos de los significados parciales de Derivada.

Tabla 2. Frecuencia y porcentajes por tipo de configuraciones cognitivas activadas en T1 y T2

\begin{tabular}{lcccc}
\hline \multicolumn{1}{c}{\begin{tabular}{c} 
Tipo de configuración \\
\multicolumn{1}{c}{ cognitiva }
\end{tabular}} & \multicolumn{2}{c}{ Frecuencias por tarea } & \multicolumn{2}{c}{ Porcentajes por tarea } \\
T1 & T2 & T1 & T2 \\
\hline Recta tangente & 54 & 33 & $60 \%$ & $37 \%$ \\
Variación/razón de cambio & 11 & 16 & $12 \%$ & $18 \%$ \\
Como límite & 5 & 11 & $6 \%$ & $12 \%$ \\
Variación/velocidad & 5 & 8 & $6 \%$ & $9 \%$ \\
Continuidad & 4 & 0 & $4 \%$ & $0 \%$ \\
Obtención de máximos y & 2 & 5 & $2 \%$ & $6 \%$ \\
mínimos & 3 & 4 & $3 \%$ & $4 \%$ \\
Otras respuestas & 6 & 13 & $7 \%$ & $14 \%$ \\
Sin respuesta & & & & \\
\hline
\end{tabular}

Aunque se podría esperar que se concibiera a la derivada como un límite — por su definición formal de la cual se derivan las reglas de derivación y que, además, se le dedica tiempo para identificar casos de funciones en las que los límites unilaterales no tienen el mismo valor (como es el caso de T7 y T8) - los alumnos hicieron una mayor referencia a otros significados $(94 \%$ y $88 \%$ de las respuestas de T1 y T2, respectivamente). En particular la asociación explícita de la derivada con la existencia de una recta tangente a la curva (la gráfica de la función) fue el caso más reportado.

Esto es posiblemente un indicador de que, en el esquema mental de los alumnos, existe una presencia de la representación gráfica de los significados (a pesar de que prácticamente no hubo este tipo de representaciones en las respuestas de estas tareas). Sin embargo, la aparición de este tipo de configuración cognitiva tampoco implicó que las respuestas fueran del todo correctas, pues se evaluó que sólo el $9 \%$ de los alumnos utilizaron representaciones gráficas y además contestaron apropiadamente. Y solo el 
$1 \%$ de ellos contestó correctamente ambas tareas y evidenció una configuración cognitiva en relación con la pendiente de la recta tangente. Además, apareció con cierta frecuencia la confusión entre lo que es la pendiente de la recta tangente a la curva y la misma recta tangente, como en la siguiente respuesta de un alumno:

\section{A1-22 Es la recta tangente a la función en el punto.}

La relación con el mundo físico (las configuraciones cognitivas vinculadas con variaciones y velocidades) tiene una presencia también por encima de la relacionada con los límites (17\% y $26 \%$ en T1 y T2, respectivamente). Esto es consistente con el hecho de que vincular la Derivada con fenómenos del mundo físico es una manera cercana para el alumno que vive en ese entorno físico.

\subsection{T3: Cálculo de derivada de función en un punto a partir de una gráfica}

Esta tarea (adaptada de Kendal y Stacey, 2001) consistió en movilizar el significado parcial asociado a la pendiente de la recta tangente con este enunciado:

T3: La gráfica corresponde a un "zoom" de la curva de una función. La recta tangente en el punto $A$ de la función $h(x)$ también se muestra. Encuentre el valor de la derivada de $h(x)$ en A.

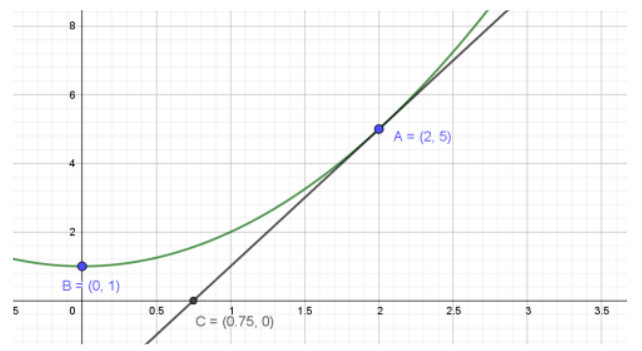

Figura 1. Gráfica proporcionada en T3

Se esperaba que los participantes consideraran los puntos $A$ y $C$ (que estan sobre la recta tangente) y únicamente calcularan la pendiente de la recta para presentarla como la respuesta. En la Tabla 3 se presenta un resumen de los tipos de respuestas donde se ve que el $18 \%$ de los participantes respondieron con un procedimiento parecido al que se esperaba, aunque sólo 10 casos $(11 \%)$ proporcionaron una respuesta correcta.

Tabla 3. Resumen de los tipos de respuesta en T3

\begin{tabular}{|c|c|c|c|c|c|}
\hline \multirow[b]{2}{*}{ Tipo de respuesta } & \multirow[b]{2}{*}{ Frecuencia } & \multirow[b]{2}{*}{ Porcentaje } & \multicolumn{3}{|c|}{ Porcentajes de respuestas por tipo } \\
\hline & & & Incorrectas & $\begin{array}{l}\text { Parcialmente } \\
\text { correctas }\end{array}$ & Correctas \\
\hline Ecuación de la recta* & 33 & $37 \%$ & $58 \%$ & $30 \%$ & $12 \%$ \\
\hline $\begin{array}{l}\text { Obtención de regla de corres- } \\
\text { pondencia y derivarla }\end{array}$ & 22 & $24 \%$ & $14 \%$ & $50 \%$ & $36 \%$ \\
\hline $\begin{array}{l}\text { Procedimiento aritmético de } \\
\text { obtención de derivada }\end{array}$ & 16 & $18 \%$ & $6 \%$ & $31 \%$ & $63 \%$ \\
\hline $\begin{array}{l}\text { Procedimiento algebraico } \\
\text { (fórmula planteada sin resol- } \\
\text { verse o calcularse) }\end{array}$ & 3 & $3 \%$ & $67 \%$ & $33 \%$ & $0 \%$ \\
\hline Referencia a gráfica original & 3 & $3 \%$ & $100 \%$ & $0 \%$ & $0 \%$ \\
\hline $\begin{array}{l}\text { Respuesta sin información } \\
\text { adicional }\end{array}$ & 2 & $2 \%$ & $50 \%$ & $50 \%$ & $0 \%$ \\
\hline Sin respuesta & 11 & $12 \%$ & -- & -- & -- \\
\hline
\end{tabular}

* Un alumno proporcionó la ecuación de la recta e intentó calcular un límite considerando a la recta como si fuese la función original. 
El $26 \%$ de respuestas fueron consideradas correctas. El tipo de respuesta con mayor número de respuestas correctas se enfocó en calcular la pendiente de la recta tangente y utilizar la fórmula proporcionada en los cursos de geometría analítica. Si bien existió en las tareas anteriores casos en los que verbalmente los estudiantes equiparaban erróneamente derivada y recta tangente, no con su pendiente, aquí no hubo ninguna respuesta que señalara a la recta como respuesta; sí hubo alumnos que obtuvieron la ecuación de la recta tangente y la usaron como respuesta o la evaluaron (con $x=2$ ) para obtener un valor numérico $(37 \%)$. Al enfrentarse a una representación gráfica no se acepta a la representación geométrica de una recta como derivada de una función (idea poco refinada que se evidenció en T1 y T2), sino que ésta última debe ser un objeto representado en el registro de representación numérico o algebraico y eso se ve en ese porcentaje de respuestas que es el mayor tipo de respuestas. El $58 \%$ de este tipo resultan respuestas incorrectas, ya sea por errores aritméticos o algebraicos, o porque se plantea como respuesta a la tarea (incluso aunque se haya calculado la pendiente de la recta como parte del proceso de obtención de la ecuación de la recta).

En resumen, es consistente la idea falsa expresada verbalmente de que la derivada es la recta tangente, sólo que debe ser expresada de manera simbólica y no geométrica. Esto queda ejemplificado por la respuesta de A1-19 que aparece en la Figura 2.

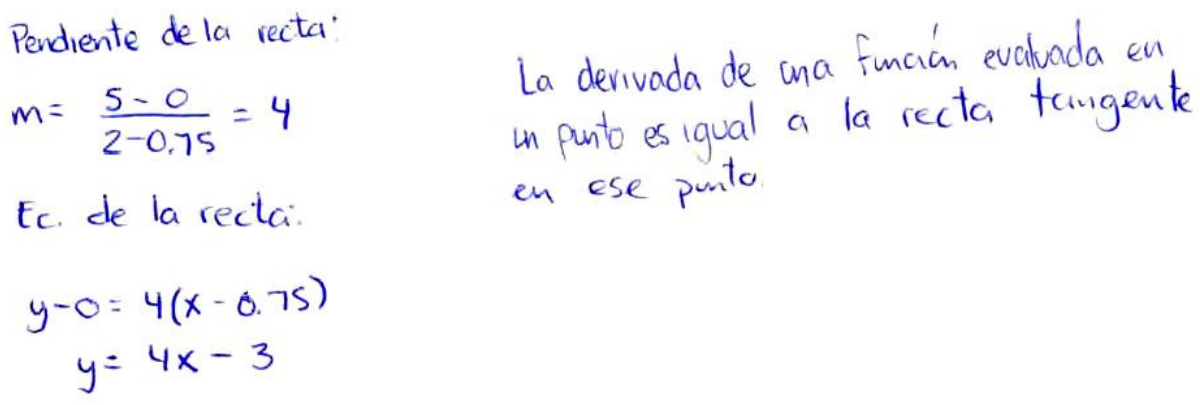

Figura 2. Respuesta de A1-19 en T3

Otra respuesta común fue la obtención de la regla de correspondencia de la función para derivarla (24 \% de casos). Pareciera que la representación gráfica de la función y de la recta tangente no les fuera útil o suficiente a los alumnos, por lo que necesitaron una transformación al registro semiótico algebraico para así hacer las manipulaciones y las conversiones necesarias hasta la respuesta. Este trabajo matemático que da preferencia al registro algebraico no es exclusivo de estudiantes y está presente en el trabajo matemático de profesores. Vargas et al. (2020) señalan la tendencia y confort en trabajar en el registro algebraico para encontrar la función original y luego derivarla, y de allí sacar conclusiones.

Finalmente, señalamos el caso en que hubo referencias directas a la gráfica como tal $(3 \%)$ y que en general fueron respuestas incorrectas. Estas prácticas evidencian que el significado de derivada se confunde con el de otros objetos matemáticos. Un ejemplo se ve en la Figura 3; A3-04 vincula la derivada con la gráfica de la función a la que se ha aplicado una reflexión, la cual es coherente con su respuesta en T2. 
A3-04 la función derivada es aquella que de manera gráfica es un reflejo de la función original

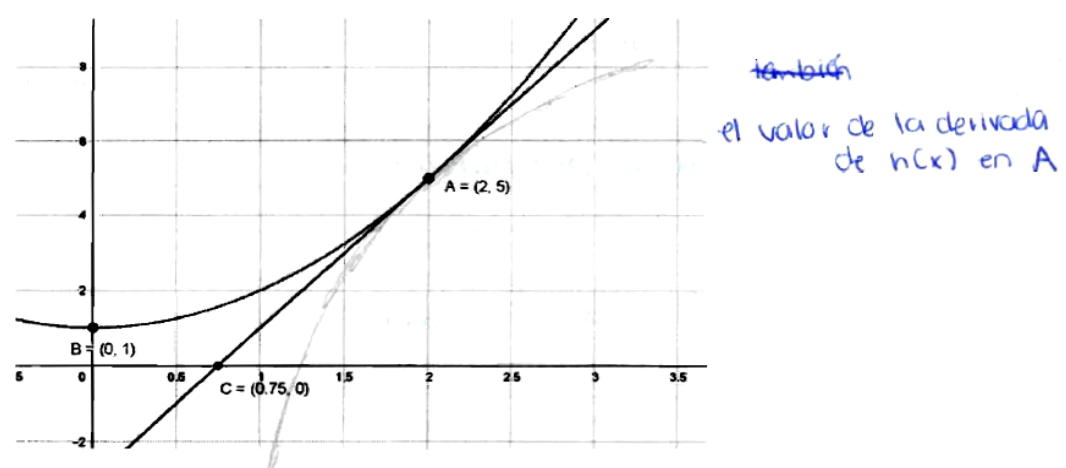

Figura 3. Respuesta de A3-04 en T3

\subsection{T4: Estimación de derivada de una función a partir de una tabulación}

Esta tarea (adaptada de Kendal y Stacey, 2001) buscó movilizar el significado parcial asociado a las variaciones mediante el cálculo de una estimación de la derivada de una función en un punto considerando una representación semiótica tabular:

T4: En la tabla se muestran valores de la función $m(x)$ cercanos al valor de $x=2$. Encuentra la mejor estimación de la derivada de $m(x)$ en $x=2$.

Tabla 4. Tabla proporcionada en T4

\begin{tabular}{cccccccccccc}
\hline$x$ & 1.95 & 1.96 & 1.97 & 1.98 & 1.99 & 2 & 2.01 & 2.02 & 2.03 & 2.04 & 2.05 \\
$m(x)$ & 2.8 & 2.84 & 2.88 & 2.92 & 2.96 & 3 & 3.04 & 3.08 & 3.12 & 3.16 & 3.2 \\
\hline
\end{tabular}

Se esperaba que los alumnos consideraran la pareja intermedia de valores $(2,3)$, que incluía el valor solicitado, y calcularan la razón de incrementos $\frac{\Delta m}{\Delta x}$ tomando los valores vecinos para estimar la derivada, pero esta práctica no fue la más común (la Tabla 5 resume tipos de respuesta). Sólo el $9 \%$ realizaron un procedimiento similar al esperado, prácticamente de tipo aritmético, mediante fórmulas de cursos de bachillerato y tomando incrementos en la variable independiente y en la función.

El tipo de respuesta más común (31\%) es buscar una regla de correspondencia que permita realizar un algoritmo de derivación, es decir, un proceso de transformación semiótica de un registro tabular a uno algebraico. Prácticamente el total de participantes que respondieron de esta manera consideraron que la variación en los datos era lineal (lo cual fue así) y, por tanto, obtuvieron una regla de correspondencia mediante las fórmulas de cursos de geometría analítica (véase Figura 4). 
Tabla 5. Resumen de los tipos de respuesta en T4

\begin{tabular}{|c|c|c|c|c|c|}
\hline \multirow[b]{2}{*}{ Tipo de respuesta } & \multirow[b]{2}{*}{ Frecuencia } & \multirow[b]{2}{*}{ Porcentaje } & \multicolumn{3}{|c|}{ Porcentajes de respuestas por tipo } \\
\hline & & & Incorrectas & $\begin{array}{l}\text { Parcialmente } \\
\text { correctas }\end{array}$ & Correctas \\
\hline $\begin{array}{l}\text { Obtención de regla de corres- } \\
\text { pondencia y derivarla* }\end{array}$ & 28 & $31 \%$ & $14 \%$ & $25 \%$ & $61 \%$ \\
\hline $\begin{array}{l}\text { Procedimiento aritmético de } \\
\text { obtención de respuesta }\end{array}$ & 8 & $9 \%$ & $12 \%$ & $25 \%$ & $63 \%$ \\
\hline $\begin{array}{l}\text { Procedimiento algebraico } \\
\text { (fórmula planteada sin resol- } \\
\text { verse o calcularse) }\end{array}$ & 1 & $1 \%$ & $100 \%$ & $0 \%$ & $0 \%$ \\
\hline Obtención de gráfica & 7 & $8 \%$ & $72 \%$ & $14 \%$ & $14 \%$ \\
\hline Uso de la razón $\frac{m(x)}{m^{\prime}(x)}$ & 4 & $4 \%$ & $100 \%$ & $0 \%$ & $0 \%$ \\
\hline Cálculo de límite & 3 & $3 \%$ & $100 \%$ & $0 \%$ & $0 \%$ \\
\hline $\begin{array}{l}\text { Obtención de ecuación de una } \\
\text { recta }\end{array}$ & 2 & $2 \%$ & $50 \%$ & $50 \%$ & $0 \%$ \\
\hline $\begin{array}{l}\text { Referencia a la misma función } \\
m(x)\end{array}$ & 2 & $2 \%$ & $100 \%$ & $0 \%$ & $0 \%$ \\
\hline Otras respuestas & 2 & $2 \%$ & $100 \%$ & $0 \%$ & $0 \%$ \\
\hline $\begin{array}{l}\text { Respuesta sin información } \\
\text { adicional }\end{array}$ & 3 & $3 \%$ & $67 \%$ & $0 \%$ & $33 \%$ \\
\hline Sin respuesta & 30 & $33 \%$ & -- & -- & -- \\
\hline
\end{tabular}

* Hubo un caso de alumno que obtuvo una regla de correspondencia, pero no intentó derivarla.

\begin{tabular}{rl|l|l|l|l|l|l|l|l|l|l|l|}
\hline$x$ & 1.95 & 1.96 & 1.97 & 1.98 & 1.99 & 2 & 2.01 & 2.02 & 2.03 & 2.04 & 2.05 \\
\hline$m(x)$ & 2.8 & 2.84 & 2.88 & 2.92 & 2.96 & 3 & 3.04 & 3.08 & 3.12 & 3.16 & 3.2 \\
\hline \multicolumn{1}{c}{$m(x)$} & $=4 x-5$ \\
$m^{\prime}(x)=4$ & $m=\frac{2.84-2.8}{1.96-1.90}=\frac{2.88-2.84}{1.97-1.96}$ \\
$m^{\prime}(2)=4$ & $\therefore$ es cha rect9 \\
$y-3$ & $=4(x-2)$ \\
$y$ & $=4 x-5$
\end{tabular}

Figura 4. Respuesta de A1-05 en T4

El uso de una gráfica (que algunos de ellos elaboraron) no garantiza que se proporcionará una respuesta correcta. Tal es el caso de los siete alumnos $(8 \%)$ que básicamente recurrieron al registro semiótico gráfico al elaborar la representación gráfica de la función $m(x)$, pero sólo uno de ellos proporcionó una respuesta correcta. Sin embargo, este proceso de transformación de una representación tabular a la gráfica, como apoyo al proceso de resolución, fue utilizado por otros cinco alumnos, por lo que el $13 \%$ de participantes lo utilizaron como apoyo o medio para responder la tarea.

Tres participantes recurrieron a la noción de límite para tratar de resolver la tarea. Aunque se les pidió una estimación del valor de la Derivada, al parecer buscaron la solución exacta haciendo referencia al hecho de que la definición formal de derivada (vinculado con su significado parcial más complejo) está basada en la noción de límite. 
Incluso uno (Figura 5) buscó los límites unilaterales. En esos tres casos las respuestas fueron incorrectas porque se evidenció un fenómeno que se comenta a continuación.

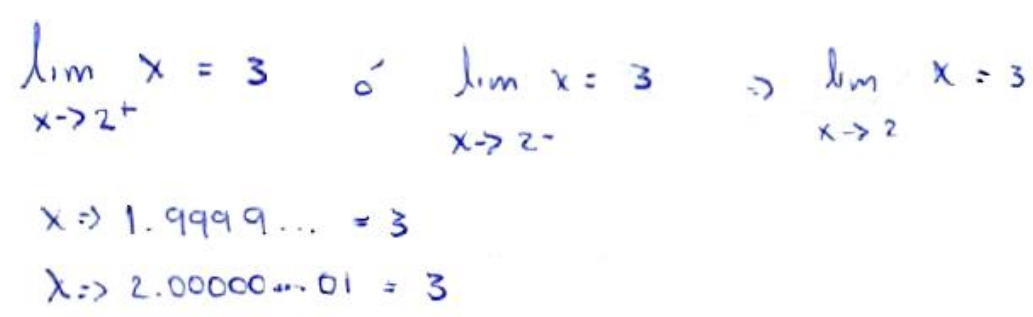

Figura 5. Respuesta de A3-10 en T4

En cinco casos se evidenció una confusión entre la función original $(m(x))$ y su derivada (que es lo que se pidió estimar), de tal manera que el tratamiento de ambos objetos fue equiparado y se proporcionaron respuestas basadas en la función, aunque se expresara que era la Derivada (véanse la misma Figura 5 y la Figura 6 por ejemplo).

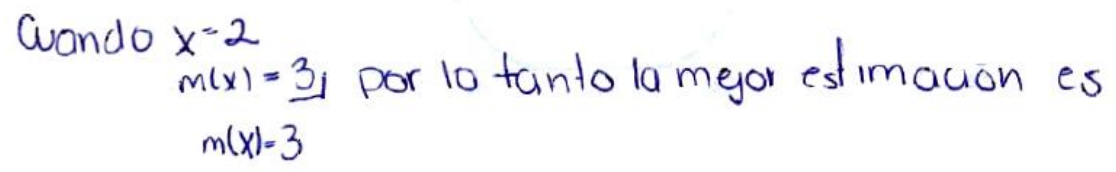

Figura 6. Respuesta de A3-18 en T4

\subsection{T6: Identificación de variaciones de la función derivada a partir de la re- presentación gráfica de la función original}

Esta tarea (adaptada de Habre y Abboud, 2006) pretendió activar los significados parciales de variaciones y máximos y mínimos de la función y relacionarlo con la función derivada. La tarea fue la siguiente:

T6: Considera la siguiente gráfica de una función $f(x)$ :

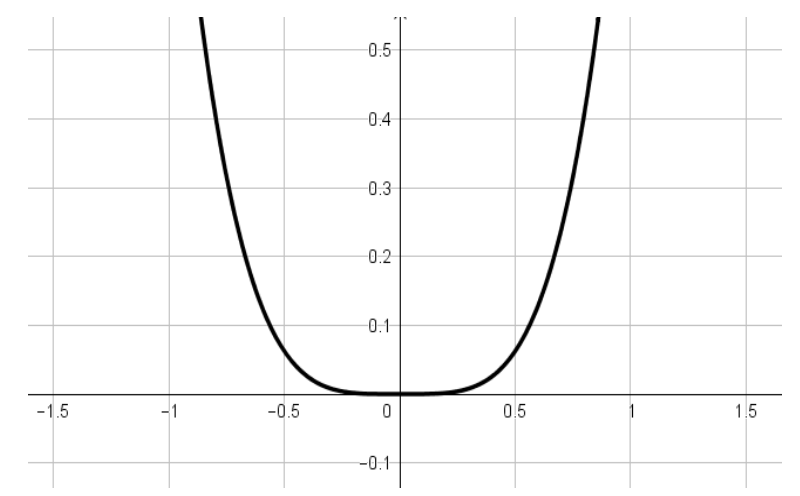

Figura 7. Gráfica de la función $f(x)$ en T6

a) Identificar los intervalos donde la función derivada de $f(x)$ es positiva y donde es negativa.

b) Identificar los intervalos donde la función derivada de $f(x)$ es creciente y donde es decreciente.

Se esperaba que los alumnos, para dar respuesta al inciso a), hicieran un tratamiento del comportamiento de la función a través de su gráfica e identificaran que en la mitad izquierda de la curva la función es decreciente y, por tanto, la función derivada toma valores negativos, mientras que en la mitad derecha la función es creciente, por 
lo que la derivada toma valores positivos. Para el inciso b) se esperaba que se identificara que, dado que la derivada pasa de valores negativos hacia positivos, se viera que es creciente en todo su dominio. En ambas identificaciones (Tablas 6 y 7) la mayoría de participantes usaron la representación gráfica para responder.

Tabla 6. Resumen de los tipos de respuesta en T6a

\begin{tabular}{|c|c|c|c|c|c|}
\hline \multirow[b]{2}{*}{ Tipo de respuesta } & \multirow[b]{2}{*}{ Frecuencia } & \multirow[b]{2}{*}{ Porcentaje } & \multicolumn{3}{|c|}{ Porcentajes de respuestas por tipo } \\
\hline & & & Incorrectas & $\begin{array}{c}\text { Parcialmente } \\
\text { correctas }\end{array}$ & Correctas \\
\hline $\begin{array}{l}\text { Referencia a registro } \\
\text { semiótico gráfico }\end{array}$ & 75 & $83 \%$ & $28 \%$ & $9 \%$ & $63 \%$ \\
\hline $\begin{array}{l}\text { Obtención de regla de } \\
\text { correspondencia y } \\
\text { derivarla }\end{array}$ & 4 & $5 \%$ & $25 \%$ & $25 \%$ & $50 \%$ \\
\hline $\begin{array}{l}\text { Uso de expresiones } \\
\text { simbólicas }\end{array}$ & 1 & $1 \%$ & $100 \%$ & $0 \%$ & $0 \%$ \\
\hline Otras respuestas & 1 & $1 \%$ & $100 \%$ & $0 \%$ & $0 \%$ \\
\hline $\begin{array}{l}\text { Respuesta sin } \\
\text { información adicional }\end{array}$ & 2 & $2 \%$ & $50 \%$ & $0 \%$ & $50 \%$ \\
\hline Sin respuesta & 7 & $8 \%$ & -- & -- & -- \\
\hline
\end{tabular}

Tabla 7. Resumen de los tipos de respuesta en T6b

\begin{tabular}{lccccc}
\hline \multicolumn{1}{c}{ Tipo de respuesta } & Frecuencia & Porcentaje & $\begin{array}{c}\text { Porcentajes de respuestas por tipo } \\
\text { Incorrectas }\end{array}$ & $\begin{array}{c}\text { Parcialmente } \\
\text { correctas }\end{array}$ & Correctas \\
\hline $\begin{array}{l}\text { Referencia a registro } \\
\text { semiótico gráfico }\end{array}$ & 72 & $80 \%$ & $25 \%$ & $50 \%$ & $18 \%$ \\
$\begin{array}{l}\text { Obtención de regla de co- } \\
\text { rrespondencia y derivarla }\end{array}$ & 2 & $2 \%$ & $0 \%$ & $0 \%$ & $100 \%$ \\
$\begin{array}{l}\text { Uso de "propiedades" } \\
\text { Sin respuesta }\end{array}$ & 4 & $4 \%$ & $50 \%$ & $25 \%$ & $25 \%$ \\
\hline
\end{tabular}

Aunque no hubo casos en que se diera una justificación donde se vincularan explícitamente las propiedades de la derivada con la forma de la gráfica proporcionada, se tuvo que la mayoría de las respuestas ( $83 \%$ y $80 \%$, respectivamente) se consideró directamente la información gráfica para responder. En algunos casos ese uso de la representación gráfica, y junto la relación de la derivada con la recta tangente a la curva, se hizo patente como es el caso que se muestra en la Figura 8.

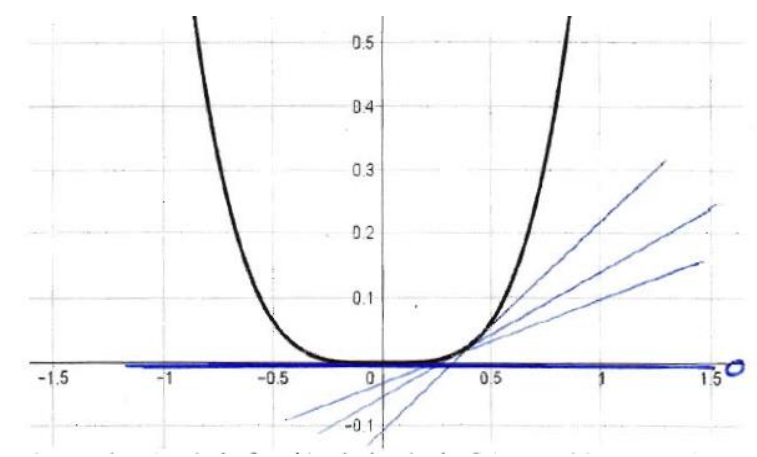

Figura 8. Trazos auxiliares que A2-06 realizó en T6 
La relación entre representación gráfica y objetos matemáticos tiene preponderancia hasta confundir en las descripciones a las diferentes representaciones semióticas con los objetos en sí, como se mencionó en el caso de T3. De manera explícita, se presentó en la siguiente respuesta donde las funciones involucradas (la original y su derivada) se equiparán con objetos geométricos (parábola y recta) que son representaciones (un proceso incorrecto de transformación entre registros):

A2-20 porque $f(x)=$ parabola por lo tanto $f^{\prime}(x)$ es una recta

Aunque la información proporcionada por esta vía puede ser útil en ocasiones, en otras limita, pues por la misma forma de la gráfica proporcionada el $21 \%$ de las respuestas (de ambas partes de la tarea) se restringió a intervalos en el dominio que estaban acotados por la imagen proporcionada (entre -1 y 1). Además, en ocasiones la información proporcionada por el registro gráfico y los conflictos cognitivos presentes en la noción de derivada llevó a sacar conclusiones erróneas a partir de la gráfica dada. Un ejemplo corresponde a que los participantes describieron en realidad el comportamiento de la función original guiándose por la gráfica proporcionada:

T6a: A3-24 $f(x)$ es positiva en el intervalo $(-\infty, \infty)$.

T6b: A3-24 El crecimiento en el intervalo $(0, \infty)$ Es decreciente en el intervalo $(-\infty, 0)$

Este fenómeno ocurrió en el $10 \%$ de los casos del inciso a) de la tarea, pero fue la razón por la que en el inciso b) la mayoría de respuestas fue considerada parcialmente correcta: 46 de los 72 estudiantes $(64 \%)$ consideró que la derivada decrecía y luego crecía, tal como ocurre con la gráfica de la función original que se proporcionó.

La estrategia de obtener una regla de correspondencia y derivarla, es decir, hacer una conversión entre registros de lo gráfico a lo algebraico para aplicar un procedimiento algorítmico y así realizar el análisis solicitado, se dio entre el $2 \%$ y $5 \%$ de los casos (hubo dos casos adicionales cuya estrategia se basó principalmente en información gráfica, pero con cierta confirmación al obtener una regla de correspondencia y derivarla). Esto permitió una tasa de éxito del $67 \%$ en ambas partes de la tarea, aunque se supuso que la gráfica correspondía a una función cuadrática.

Hubo casos (4\%) en que los alumnos presentaron sus respuestas apelando a "propiedades" que evidencian un conflicto cognitivo con el significado de referencia y que se ejemplifican en respuestas proporcionadas a T6b, como las siguientes:

A3-12 Una derivada indica si la curva es creciente o decreciente dependiendo signo, pero una derivada no tiene esa propiedad.

A2-08 Creciente, cuando de derecha a izquierda, la función va al infinito positivo. Decreciente, cuando de derecha a izquierda, la función va al infinito negativo.

Pareciera incluso que A3-12 considera que la derivada es un objeto matemático que se obtiene como un resultado (tal como se hace con una operación aritmética) pero que no se puede ampliar para ser considerada una función.

\subsection{T7: Representación de función derivada a partir de la gráfica de una fun- ción a trozos}

Esta tarea (adaptada de Montoya et al., 2017) consideró movilizar los significados parciales de la derivada relativos a rectas tangentes y límites, tomando representaciones semióticas de los registros gráfico y algebraico para su resolución. 
T7: Dada la representación gráfica de la función $g(x)$, representa la función derivada de $g(x)$.

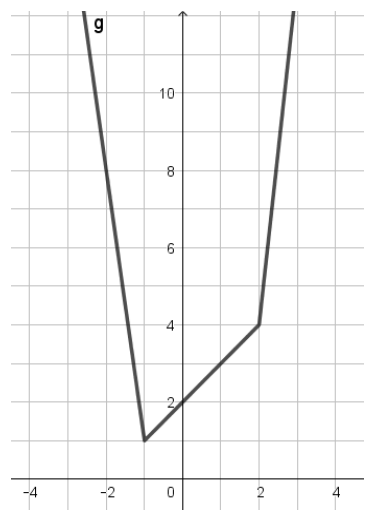

Figura 9. Gráfica de la función $g(x)$ en T7

En esta tarea no se especificó el registro de representación de la derivada. Se esperaba que los alumnos identificaran la pendiente de los trozos de la gráfica y así presentaran la respuesta considerando el caso de los puntos donde cambiaban las reglas de correspondencia. La Tabla 8 presenta un resumen de los tipos de respuesta.

Tabla 8. Resumen de los tipos de respuesta en $T 7$

\begin{tabular}{lccccc}
\hline \multicolumn{1}{c}{ Tipo de respuesta } & Frecuencia & Porcentaje & Incorrectas & $\begin{array}{c}\text { Porcentajes de respuestas por tipo } \\
\text { Parcialmente } \\
\text { correctas }\end{array}$ & Correctas \\
\hline $\begin{array}{l}\text { Uso de registro } \\
\text { semiótico gráfico }\end{array}$ & 23 & $26 \%$ & $65 \%$ & $27 \%$ & $9 \%$ \\
$\begin{array}{l}\text { Obtención de regla de } \\
\text { correspondencia y } \\
\text { derivarla }\end{array}$ & 22 & $24 \%$ & $23 \%$ & $59 \%$ & $18 \%$ \\
$\begin{array}{l}\text { Obtención de regla de } \\
\text { correspondencia sin } \\
\text { derivarla }\end{array}$ & 9 & $10 \%$ & $100 \%$ & $0 \%$ & $0 \%$ \\
$\begin{array}{l}\text { Cálculo aritmético de } \\
\text { pendiente }\end{array}$ & 2 & $2 \%$ & $0 \%$ & $100 \%$ & $0 \%$ \\
$\begin{array}{l}\text { Consideración de que } \\
\text { no es función }\end{array}$ & 1 & $1 \%$ & $100 \%$ & $0 \%$ & $0 \%$ \\
$\begin{array}{l}\text { Respuesta sin infor- } \\
\text { mación adicional }\end{array}$ & 1 & $1 \%$ & $100 \%$ & $0 \%$ & $0 \%$ \\
\begin{tabular}{l} 
Sin respuesta \\
\hline
\end{tabular} & 32 & $36 \%$ & -- & -- & -- \\
\hline
\end{tabular}

Esta tarea fue más difícil para los participantes que las anteriores, lo que se evidenció con que más de un tercio $(36 \%)$ de ellos no la contestaron. Al ser una función a trozos y no tener la regla de correspondencia, poco más de la cuarta parte $(26 \%)$ recurrió al uso de la información proporcionada por la gráfica, aunque la mayor parte de ellos $(65 \%)$ respondió de manera incorrecta. Seis de ellos determinaron que no era derivable la función porque era por trozos o porque tiene "picos":

\section{A2-02 Una gráfica con picos no tiene derivada}

Este tipo de respuesta evidencia la generalización de casos particulares con base en la información gráfica. Sólo un caso de los seis que se indican hace una mención que permite pensar que el alumno ha llevado a cabo un proceso de transformación entre los registros gráfico y algebraico al vincularlo con la idea de límite y de límite unilateral: 
A1-23 La funcion $g(x)$ no cuenta con derivada ya que de la grafica se observa en los puntos donde cambia que los limites cuando se tiende por la derecha e izquierda no es igual. puede ser una funcion por partes.

Como no se dijo cómo representarlo, los participantes representaron la función resultante utilizando dos registros semióticos: el algebraico y el gráfico. 17 alumnos utilizaron sólo el registro algebraico; doce, el registro gráfico y siete dieron una respuesta usando el registro algebraico respaldado con el gráfico. Un grupo de participantes resolvió la tarea realizando conversión entre registros de representación; tomaron información gráfica y buscaron reglas de correspondencia que les permitiera aplicar las fórmulas de derivación. El $24 \%$ de participantes hicieron esto y, aunque sólo el $18 \%$ de ellos terminaron proporcionando una respuesta correcta, este nivel de logro fue mayor al $9 \%$ de los que utilizaron la información gráfica para resolver.

El $10 \%$ de casos se enfocaron en obtener la regla de correspondencia de la función (varias veces de manera parcial), sin proceder a derivar. Pareciera que los alumnos están enfocados en describir la función original y no la función derivada. Un caso relacionado con esto es la respuesta de A2-07, centrada en describir el comportamiento de la función a partir de propiedades que seguramente se vieron en clase (Figura 10).

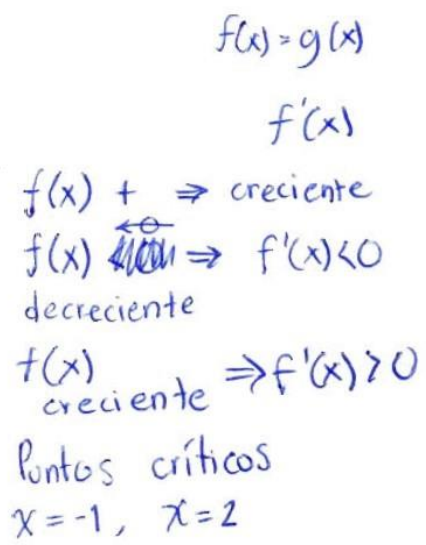

Figura 10. Respuesta de A2-07 en T7

\section{Comentarios finales sobre los significados de derivada}

Si curricularmente se piensa, sobre todo, en el significado parcial vinculado con la noción de límite (Pino-Fan et al., 2013), lo que se observó en este estudio es que el significado parcial predominante de derivada está vinculado con las rectas tangentes a la gráfica de la función original. Se observó en T3 que, si bien no se aceptó a la recta de la gráfica como derivada de la función en el punto dado y que se esperaba un objeto expresado en el registro semiótico algebraico, una cantidad apreciable de participantes aceptó la ecuación de una recta (que es una representación de una línea recta en un registro semiótico algebraico) como la derivada.

De igual forma, en las primeras tareas hubo presencia del significado parcial vinculado con la razón de cambio, aunque en menor medida que el vinculado con las rectas tangentes. Las tasas de respuestas correctas en T3 y T4 fueron similares, considerando que T3 estuvo ligada al significado de rectas tangentes y T4 al de razón de cambio. Sin embargo, las referencias al significado parcial de las rectas tangentes siguieron apareciendo de manera reiterativa en el desarrollo de las otras tareas. 
Se observó que, ante la situación de proporcionar a los alumnos información en los registros semióticos gráfico y tabular, hubo una preferencia por utilizar el gráfico, manifestándose problemas al momento de realizar conversiones de un registro a otro.

Hubo un cierto porcentaje de participantes que hicieron (correcta o incorrectamente) conversiones hacia el registro algebraico, y tratamientos en este mismo registro, que les permitió afrontar la tarea con técnicas analíticas. Sin embargo, estas prácticas tuvieron la limitante de que si la función proporcionada no era reconocida (o conocida) por el alumno entonces no se podía llevar a cabo y todo se centraba en la información proporcionada (mayormente gráfica).

En este sentido, ocurrió algo semejante a lo que se ha visto en el aprendizaje de otras áreas de las Matemáticas (e. g. Scaglia y Moriena, 2005, para geometría) y que también mencionan Habre y Abboud (2006): los alumnos utilizan prototipos ya construidos en su desarrollo académico (y personal) para abordar nuevas situaciones. Cada prototipo es el ejemplar que es reconocido como el más idóneo por los individuos (Kleiber, 1995, p. 48). Esto quiere decir que los prototipos provienen de los significados personales de cada individuo y tienen la desventaja de que su carácter de "idóneo" es asignado por el significado del individuo y las posibilidades que tiene de proporcionar información o utilidad en ciertas condiciones conocidas previamente. Así que, si bien la estrategia de considerar prototipos puede ayudar a tener éxito al abordar algunas tareas, también se puede constituir en un obstáculo, porque puede proporcionar información errónea y llevar a conclusiones limitadas o equivocadas.

Los alumnos supusieron que las curvas de T3 y T6 eran gráficas de funciones cuadráticas, por lo que las obtuvieron y pudieron derivar; abordaron T4 asumiendo una relación lineal entre variables, y en T7 consideraron que la gráfica estaba constituida por dos semirrectas y un segmento de recta. Todos estos supuestos fueron correctos, más por el diseño de las tareas que por coincidencia. Como dicen Habre y Abboud (2006, p. 63), en ausencia de una definición algebraica de una función, los estudiantes sólo pueden razonar pensando en funciones como $y=x^{2}$, o polinomiales en general.

Si los procesos de conversión entre registros semióticos no son eficaces, el aprendizaje y la construcción de significados personales se verán limitados. Por lo que entonces se requiere hacer énfasis en estas acciones e incluso cuidar el lenguaje verbal utilizado por parte del profesor, como lo identifica Park (2015, p. 249):

Los instructores dicen "secante" cuando quieren decir "pendiente de la secante" y "tangente" cuando quieren decir "pendiente de la tangente". Dibujan gráficas para ilustrar la derivada en un punto, pero ya no usan gráficas cuando definen la derivada de una función.

En el ámbito académico de la formación de ingenieros, y en su mismo ámbito profesional, existen muchos fenómenos y situaciones donde los modelos matemáticos no aluden necesariamente a una relación dinámica entre variables (e. g. el grado de deformación de una viga en función de sus medidas de largo, ancho y algo). Aunque el modelo matemático considere funciones continuas, la situación del mundo profesional no es una función continua o algo que se pueda modificar de manera dinámica sino algo que podría cambiarse sólo si se vuelve a hacer.

Se requiere del profesor, en los cursos, una selección de tareas apropiadas, que haga referencia a una muestra representativa de los significados parciales de derivada para que se construya un significado holístico, y que aprovechen las diferentes representaciones semióticas utilizadas para expresar funciones, derivadas y objetos matemáticos asociados. En consecuencia, se considera necesario que los profesores tengan en 
cuenta la complejidad del objeto matemático que se pretende enseñar (entendida como pluralidad de significados) en sus procesos de instrucción.

Las perspectivas de este trabajo de investigación corresponden al diseño de tareas para que el estudiante futuro ingeniero construya el significado holístico de derivada. Se plantea integrar en ese diseño de tareas acciones que permitan identificar niveles de comprensión de la derivada. Así que uno de nuestros fines no está subordinado únicamente al interés de la investigación, sino al diseño de tareas para la puesta a punto de materiales para el aula, con el fin de contar con un repertorio idóneo. Asimismo, se pretende influir principalmente en el contenido curricular y en el enfoque que deben tener los cursos de cálculo en las ingenierías.

\section{Agradecimientos}

Al proyecto sabático "Estudio sobre las dificultades en la construcción de significados de la derivada por alumnos de Ingeniería", apoyado por Universidad Autónoma de Querétaro (UAQ) y Centro Interdisciplinario de Investigación y Docencia en Educación Técnica (CIIDET).

\section{Referencias}

Arcos., J. I. (2019). Una presentación de los conceptos del cálculo, en escuelas de ingeniería, no centrada en la definición de límite. El Cálculo y su Enseñanza. Enseñanza de las Ciencias y la Matemática, 12(1), 46-59.

Arcos, J. I. y Sepúlveda, A. (2014). Desarrollo conceptual del cálculo. Desarrollo histórico de los conceptos del cálculo: Una perspectiva docente. Universidad Autónoma del Estado de México.

Ariza, Á. y Llinares, S. (2009). Sobre la aplicación y uso del concepto de derivada en el estudio de conceptos económicos en estudiantes de bachillerato y universidad. Enseñanza de las Ciencias, 27(1), 121-136. https://doi.org/10.5565/rev/ensciencias.3667

Artigue, M., Batanero, C. y Kent, P. (2007). Mathematics thinking and learning at post-secondary level. En F. K. Lester (Ed.), Second Handbook of Research on Mathematics Teaching and Learning (pp. 1011-1049). NCTM e IAP.

Bingolbali, E., Monaghan, J. y Roper, T. (2007). Engineering students' conceptions of the derivative and some implications for their mathematical education. International Journal of Mathematical Education in Science and Technology, 38(6), 763-777. https://doi.org/10.1080/00207390701453579

Cuevas, O., Larios, V., Peralta, J. X. y Jiménez, A. R. (2018). Mathematical knowledge of students who aspire to enroll in engineering programs. International Electronic Journal of Mathematics Education, 13(3), 161-169. https://doi.org/10.12973/iejme/3832

Duval, R. (1993). Registres de représentation sémiotique et fonctionnement cognitif de la pensée. Annales de Didactique et de Sciences Cognitives, 5, 37-65.

Gnedenko, B. V. y Khalil, Z. (1979). The mathematical education of engineers. Educational Studies in Mathematics, 10(1), 71-83. https://doi.org/10.1007/BF00311176 
Godino, J. D., Batanero, C. y Font, V. (2007). The onto-semiotic approach to research in mathematics education. ZDM, 39(1-2), 127-135. https://doi.org/10.1007/s11858-006-0004-1

Godino, J. D., Batanero, C. y Font, V. (2019). The Onto-semiotic Approach: Implications for the prescriptive character of didactics. For the Learning of Mathematics, 39(1), 37-42.

Habre, S. y Abboud, M. (2006). Students' conceptual understanding of a function and its derivative in an experimental calculus course. Journal of Mathematical Behavior, 25(1), 57-72. https://doi.org/10.1016/j.jmathb.2005.11.004

Hitt, F. (2005). Dificultades en el aprendizaje del cálculo. En J. C. Cortés y F. Hitt (Eds.), Reflexiones sobre el aprendizaje del cálculo y su enseñanza (pp. 81-107). Morevallado Editores.

Kendal, M. y Stacey, K. (2001). The impact of teacher privileging on learning differentiation with technology. International Journal of Computers for Mathematical Learning, 6(2), 143-165. https://doi.org/10.1023/A:1017986520658

Kleiber, G. (1995). La semántica de los prototipos. Visor Libros.

Montoya, E., Páez, R. E. y Vivier, L. (2017). Les perspectives de localité dans le travail en analyse. En I. Gómez et al. (Eds.), Proceedings Fifth ETM Symposium (pp. 79-94). University of Western Macedonia.

Orts, A., Llinares, S. y Boigues, F. J. (2016). Elementos para una descomposición genética del concepto de recta tangente. Avances de Investigación en Educación Matemática, 10, 111-134.

Park, J. (2015). Is the derivative a function? If so, how do we teach it? Educational Studies in Mathematics, 89(2), 233-250. https://doi.org/10.1007/s10649-015-9601-7

Pino-Fan, L. R., Castro, W. F., Godino, J. D. y Font, V. (2013). Idoneidad epistémica del significado de la derivada en el currículo de bachillerato. Paradigma, 34(2), 123-150.

Pino-Fan, L. R., Font, V., Gordillo, W., Larios O. y Breda, A. (2018). Analysis of the meanings of the antiderivative used by students of the first engineering courses. International Journal of Science and Mathematics Education, 16(6), 1091-1113. https://doi.org/10.1007/s10763-017-9826-2

Pino-Fan, L. R., Godino, J. D. y Font M., V. (2011). Faceta epistémica del conocimiento didáctico-matemático sobre la derivada. Educação Matematica e Pesquisa, 13(1), 141-178.

Romo-Vázquez, A. (2014). La modelización matemática en la formación de ingenieros. Educación Matemática, 26(Especial 25 Años), 314-338.

Scaglia, S. y Moriena, S. (2005). Prototipos y estereotipos en geometría. Educación Matemática, 17(3), 105-120.

Vargas, M. F., Fernández-Plaza, J. A. y Ruiz-Hidalgo, J. F. (2020). Análisis de los argumentos dados por docentes en formación a una tarea sobre derivadas. PNA, 14 (3), 173-203. 


\section{Referencias de los autores}

Víctor Larios Osorio, Universidad Autónoma de Querétaro (México) vil@uaq.mx

Rosa Elvira Páez Murillo, Universidad Autónoma de la Ciudad de México (México) rosa.paez@uacm.edu.mx

Hugo Moreno Reyes, Centro Interdisciplinario de Investigación y Docencia en Educación Técnica (México) hmoreno@ciidet.edu.mx 


\section{Meanings on the derivative evidenced by Engineering students at a Mexican uni- versity}

Víctor Larios Osorio, Universidad Autónoma de Querétaro (México)

Rosa Elvira Páez Murillo, Universidad Autónoma de la Ciudad de México (México)

Hugo Moreno Reyes, Centro Interdisciplinario de Investigación y Docencia en Educación Técnica (México)

The teaching of Calculus has a fundamental role for the formation of engineers for it is the basis of other studies in their education. This is one of the main reasons why the study of Calculus learning and the problems on its teaching has become more and more relevant. In this sense the present paper's goal is to answer the question: What is the meaning of the mathematical object Derived for a group of future engineers? For this purpose, the Ontosemiotic Approach to Mathematical Cognition and Instruction (OSA) is used as a theoretical reference, which considers a pragmatic view of the mathematical activity in the classroom and considers the complexity of the mathematical object understood as a plurality of meanings. The methodology used describes the following steps: 1) A reconstruction of the holistic meaning of the Derived is presented, composed by nine partial meanings that have been presented throughout its historical development. 2) A questionnaire was designed with the proposition of eight tasks, in which some of the nine partial meanings are expected to be used. 3) The questionnaire was applied, and the answers of 90 Engineering students at a Mexican university were analyzed in a qualitative way (using the ontosemiotic analysis method) and in a quantitative way. As results, the meanings of the Derivative object inferred from the participants' answers are presented and it is concluded that the predominant partial meaning of the Derivative is linked to the tangent lines to the graph of the original function; also, although to a lesser extent, there was a relevant presence of the partial meaning linked to the reason for change. Likewise, it was observed a preference for using the graphic register when the information is presented between this one and the tabular one, identifying difficulties of conversions between the different semiotic registers commonly used in the courses. We end with some reflections on the tasks to be considered in the courses of calculus aimed at the training of engineers, the latter being a perspective of this research work and the work team formed. 